\title{
Complete Disappearance of A Locally Advanced Gastric Tumor Under Chemotherapy: Case Report
}

Alae Eddine El Aissaoui*, Mourad Badri, Alliou Zabeirou, Rayhana Boujarnija, Tarik souiki, Ahmed Zerhouni, Karim Ibn Majdoub, Khalid Mazaz, Imane Toughrai

Visceral Surgery Service, Hassan II University Hospital, Faculty of Medicine and Pharmacy of Fez, Sidi Mohammed Ben Abdallah University, Fez, Morocco

DOI: $10.36347 /$ sjmcr.2020.v08i04.022

| Received: 28.03.2020 | Accepted: 04.04.2020 | Published: 30.04 .2020

*Corresponding author: Alae Eddine El Aissaoui

\section{Abstract}

Gastric cancer is one of the most common cancers worldwide. Its most common histological form is adenocarcinoma. Perioperative chemotherapy is currently the reference treatment for resectable gastric adenocarcinomas, yet the surgery is the only potentially curative treatment. We report the case of a 50 year old patient, with a history of diabetes, hospitalized for epicastric pain evolving for several months in a context of unencrypted weight loss. The esogastric endoscopy revealed a large ulcero-proliferative growth in the antro-pyloric region while the anatomopathological study of the biopsy revealed an undifferentiated carcinomatous gastric process. The CT scan finds a locally advanced gastric antropyloric tumor. The patient received perioperative FLOT protocol chemotherapy with an excellent therapeutic response which has been demonstrated on the CT scan that has shown the absence of any gastric thickening or pancreatic processes. A distal subtotal gastrectomy was performed, with modified D2 (D1+) lymphadenectomy and a Billroth II Reconstruction. The anatomopathological study of the surgical piece revealed the absence of dysplasia or tumor proliferation. Postoperative recovery was uneventful and the patient received postoperative chemotherapy with a favorable evolution to date. Gastric cancer is a common tumor that requires multidisciplinary care for optimal therapeutic strategy. Perioperative chemotherapy is the reference treatment for resectable gastric adenocarcinomas; it allows the evaluation of chemical response, to have a downstaging that may facilitate surgery while getting an early systemic effect.

Keywords: Gastric Tumor Chemotherapy resectable epicastric.

Copyright @ 2020: This is an open-access article distributed under the terms of the Creative Commons Attribution license which permits unrestricted use, distribution, and reproduction in any medium for non-commercial use (NonCommercial, or CC-BY-NC) provided the original author and source are credited.

\section{INTRODUCTION}

Gastric cancer is the $4^{\text {th }}$ most common cancer in men and the $5^{\text {th }}$ in women. Surgery is the only potentially curative treatment for gastric cancer. Neoadjuvant chemotherapy is a promising strategy with good tolerance without post-operative complications.

We report the case of a patient who presented a locally advanced gastric tumor which disappeared morphologically after neo-adjuvant chemotherapy thus allowing an optimal carcinological surgical gesture.

\section{CASE RePORT}

50 year old patient with a history of type II diabetes, hospitalized for epigastric pain evolving for 4 months, with postprandial vomiting evolving in a context of unencrypted weight loss.
The clinical examination found a tenderness of the epigastrium. The lymph node areas were free and the rest of the abdominal examination was without abnormalities.

Upper gastro-intestinal endoscopy revealed a large ulcero-proliferative growth in the antro-pyloric region causing luminal stenosis. The anatomopathological study of the biopsy revealed an undifferentiated carcinomatous gastric process with suspicion of neuroendocrine origin.

The CT scann found a locally advanced gastric antropyloric tumor invading the pancreas without any secondary localization. 


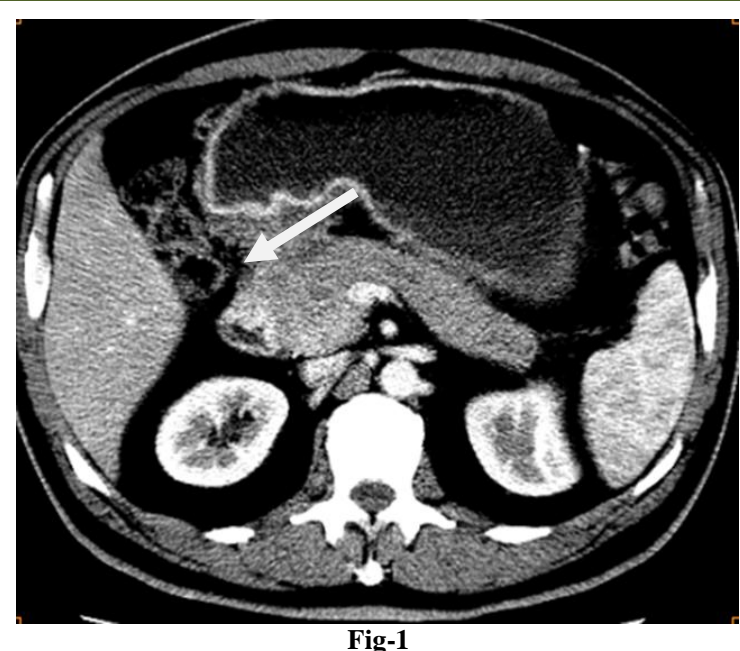

The decision of the multidisciplinary concertation meeting (RCP) was neoadjuvant chemotherapy then reassessment.

The patient received perioperative FLOT protocol chemotherapy (4 Cycles). Post-chemotherapy CT scan didn't report any suspected abdominal abnormality: absence of any gastric thickening or pancreatic processes.

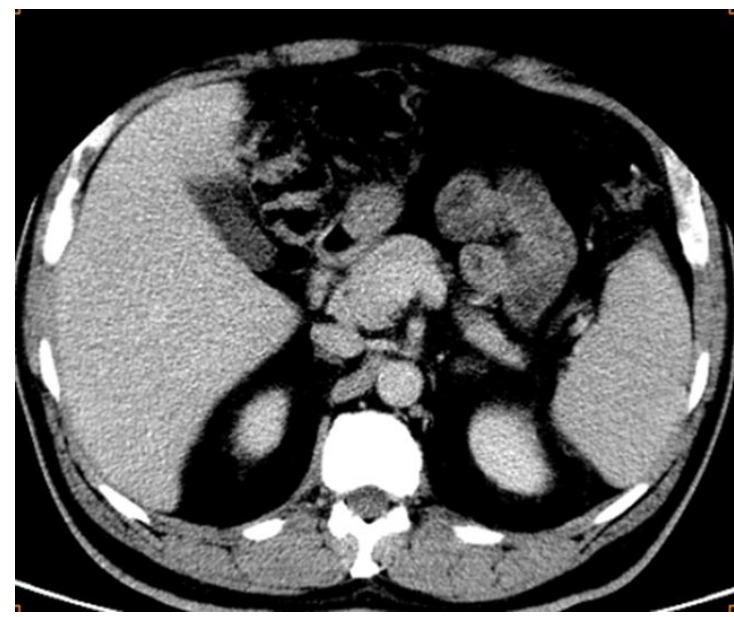

Fig-2

The decision of the secondary multidisciplinary concertation meeting was the surgery. The patient was operated having a distal subtotal gastrectomy with modified D2 (D1+) lymphadenectomy and a Billroth II reconstruction.

The anatomopathological study of the surgical piece found just a fibrous and inflammatory remodeling of the gastric mucosa with the presence of mild and focal intestinal metaplasia and the absence of dysplasia or tumor proliferation.

Postoperative recovery was uneventful and the patient was discharged from hospital 5 days later. The decision of the RCP was postoperative chemotherapy (4 cycles). The evolution is favorable to date.

\section{DisCUSSION}

Gastric cancer is a common oncologic disease witch prognosis is still poor. It ranks $4^{\text {th }}$ in cancer worldwide and is the $3^{\text {rd }}$ leading cause of cancer mortality [1].

Oeso-gastric endoscopy is essential for the positive diagnosis that allows biopsies to be performed and to measure the distance of the tumor from cardia and pylorus. According to the German guidelines S3 [2$3]$, at least eight biopsies are required for all suspected lesions. If histologic findings are negative, a new endoscopy is recommended in 2 weeks with repeated biopsies at the edge and at the center of the lesion.

Surgery makes the only treatment that can guarantee a cure for gastric cancer [4]. In the case of mucosal limited carcinoma (cT1a), the presence of lymph node metastases is extremely rare, and endoscopic resection in this context may be sufficient.

In locally advanced forms, most cancer surgeons recommend extending the resection to the surrounding organs when they are invaded:

- In a series of gastric cancer with invasion of neighboring structures, Kodama et al. [5] shows that combined monobloc resections of invaded organs offer better 5-year survival $(23 \%)$ compared to palliative resections in which invaded organs has not been reached. The conclusion is that monobloc enlarged resection should always be attempted in case of locally advanced gastric cancer

- Takeuchi et al. [6] have shown that there is no benefit of spleno-pancreatectomy associated with total gastrectomy in non-metastatic patients.

- Iriyama et al. [7] report a 5-year survival of $46 \%$ after enlarged gastrectomy to adjacent organs in $\mathrm{T} 4$ cancers.

Perioperative chemotherapy is currently the reference treatment for resectable gastric adenocarcinomas. The chemotherapy used can be done according to an ECF scheme ( 3 cures before and 3 cures after surgery) or 5-FU-cisplatin ( 2 to 3 cures before and after surgery) [8].

The FLOT-4 study shows that perioperative chemotherapy with 5-fluorouracil, leucovorine, oxaliplatin, and docetaxel (FLOT) increases the survival of stomach cancers, compared to baseline chemotherapy involving epirubicin, cisplatin, and 5 fluorouracil (or capecitabine) (ECF / ECX) [9]. It has been integrated into the French [10] and European [11] repositories, however, question remain about whether it can be used for all patients.

The MAGIC study [12] has established evidence for this perioperative approach. 503 patients with gastric cancer, esogastric or esophageal junction 
were included and then randomized to receive perioperative chemotherapy and then surgery or surgery alone. The risk of recurrence was significantly decreased and the risk of death at 5 years $(13 \%)$ in the arm with chemotherapy.

Current recommendations are clear for TNMclass cT3 and cT4a tumors that may benefit from resection at a later time. After perioperative treatment, a new complementary examination by endoscopy and thoraco-abdominal CT-scan should be performed to exclude progression under neoadjuvant treatment and confirm tumor resectability.

The postoperative chemotherapy should be performed based on the patient's general condition and tolerance to the first treatment. The prognostic relevance is indeed unclear at present $[13,14]$.

Following the surgical resection in patients without a prior treatment with an underestimated preoperative tumor stage which could be advanced or patients undergoing emergency surgery, an adjuvant treatment may be discussed.

Postoperative systemic chemotherapy has been studied in many trials, and its indication has not been retained after $\mathrm{R} 0$ resection of gastric adenocarcinoma [15].

\section{Conclusion}

Gastric cancer is a common tumor that requires multidisciplinary care right from the diagnosis in order to determine the optimal therapeutic strategy.

The advantage of neo-adjuvant chemotherapy is the ability to evaluate the response of the tumor, obtain a downstaging that may facilitate the surgery while getting an early systemic effect.

\section{REFERENCES}

1 Jemal A, Center MM, DeSantis C, Ward EM. Global patterns of cancer incidence and mortality rates and trends. Cancer Epidemiology and Prevention Biomarkers. 2010 Aug 1;19(8):1893907.

2 C Allemani T Matsuda V Di Carlo Global surveillance of trends in cancer survival 2000-14 (CONCORD-3): analysis of individual records for 37513025 patients diagnosed with one of 18 cancers from 322 population-basedregistriesin 71 countries. Lancet. 2018; (391)

3 HJ Meyer AH Hölscher F Lordick Current S3 guidelines on surgical treatment of gastric carcinoma. Chirurg. 2012 (83)

4 Takeuchi K, Tsuzuki Y, Ando T, Sekihara M, Hara T, Yoshikawa M, Ohno Y, Kuwano H. Total gastrectomywith distal pancreatectomy and splenectomy for advancedgastric cancer. J Surg Res. 2001; 101: 196-201.

5 Kodama I, Takamiya H, Mizutani K, Ohta J, Aoyagi K, Kofuji K, Takeda J, Shirouzu K. Gastrectomy with combined resection of other organs for carcinoma of the stomach with invasion to adjacent organs: clinical efficacy in a retrospective study. J Am Coll Surg. 1997; 184: 1622

6 Takeuchi K, Tsuzuki Y, Ando T, Sekihara M, Hara T, Yoshikawa M, Ohno Y, Kuwano H. Total gastrectomy with distal pancreatectomy and splenectomy for advanced gastric cancer. J Surg Res. 2001; 101: 196-201.

7 Iriyama K, Ohsawa T, Tsuchibashi T, Noji M, Miki C, Ilunga K, Suzuki H. Results of combined resection of invaded organs in patients with potentially curable, advanced gastric cancer. Eur J Surg. 1994; 160: 27-30

8 Aparicio T, Yacoub M, Karila-Cohen P, René E. Adénocarcinome gastrique: notions fondamentales, diagnostic et traitement. EMC-Chirurgie. $2004 \mathrm{Feb}$ 1;1(1):47-66.

9 Adenis A, Samalin E, Mazard T, Portales F, Mourregot A, Ychou M. Does the FLOT regimen a new standard of perioperative chemotherapy for localized gastric cancer?. Bulletin du Cancer. 2020 Jan 21;107(1):54-60.

10 Zaanan A, Bouché O, Benhaim L, Buecher B, Chapelle N, Dubreuil O, Fares N, Granger V, Lefort C, Gagniere J, Meilleroux J. Gastric cancer: French intergroup clinical practice guidelines for diagnosis, treatments and follow-up (snfge, ffcd, gercor, unicancer, sfcd, sfed, sfro). Digestive and Liver Disease. 2018 Aug 1;50(8):768-79.

11 Smyth EC, Verheij M, Allum W, Cunningham D, Cervantes A, Arnold D. Gastric cancer: ESMO Clinical Practice Guidelines for diagnosis, treatment and follow-up. Annals of oncology. 2016 Sep 1;27(suppl_5):v38-49.

12 Catalano V, Labianca R, Beretta GD, Gatta G, De Braud F, Van Cutsem E. Gastric cancer. Critical reviews in oncology/hematology. 2005 Jun 1;54(3):209-41.

13 Meyer HJ, Hölscher AH, Lordick F, Messmann H, Mönig S, Schumacher C, Stahl M, Wilke H, Möhler M. Current S3 guidelines on surgical treatment of gastric carcinoma. Der Chirurg; Zeitschrift fur alle Gebiete der operativen Medizen. 2012 Jan;83(1):31-7.

14 Smyth EC, Verheij M, Allum W, Cunningham D, Cervantes A, Arnold D. Gastric cancer: ESMO Clinical Practice Guidelines for diagnosis, treatment and follow-up. Annals of oncology. 2016 Sep 1;27(suppl_5):v38-49.

15 Michel P, Di Fiore F. Chimiothérapie adjuvante du cancer gastrique. Gastroentérologie Clinique et Biologique. 2006 Jan 1;30(1):96-101. 